\title{
Supporting Information for The Adsorption of Perfluorooctane Sulfonate onto Sand, Clay, and Iron Oxide Surfaces
}

\author{
RAMONA L. JOHNSON , AMY ANSCHUTZ ${ }^{\ddagger}$, JEAN M. SMOLEN ${ }^{\dagger}$, MATT F. \\ SIMCIK , AND R. LEE PENN ${ }^{*}$ \\ Department of Chemistry, Saint Joseph's University, 5600 City Avenue, Philadelphia, \\ PA 19131 \\ Department of Chemistry, University of Minnesota, 207 Pleasant Street SE,
} Minneapolis, MN 55455

Division of Environmental Health Sciences, School of Public Health, University of Minnesota, MMC 807, 420 Delaware Street SE, Minneapolis, MN 55455

Figures: 0; Tables: 5Atomic Charges on n-octanesulfonate and PFOS

Table 1

n-octanesulfonate Standard orientation:

\begin{tabular}{|c|c|c|c|c|c|}
\hline \multirow{2}{*}{$\begin{array}{l}\text { Center } \\
\text { Number }\end{array}$} & \multirow{2}{*}{\multicolumn{2}{|c|}{$\begin{array}{l}\text { Atomic } \\
\text { Number }\end{array}$}} & \multirow{2}{*}{$\begin{array}{r}\text { Atomic } \\
\text { Type }\end{array}$} & \multicolumn{2}{|c|}{ Coordinates (Angstr } \\
\hline & & & & $\mathbf{X}$ & $\mathbf{Z}$ \\
\hline 1 & 6 & $\mathbf{0}$ & 5.521145 & 3.956805 & .000000 \\
\hline 2 & 6 & $\mathbf{0}$ & 3.990836 & 3.875588 & .000000 \\
\hline 3 & 6 & $\mathbf{0}$ & 3.457304 & 2.436902 & .000000 \\
\hline 4 & 6 & $\mathbf{0}$ & 1.926005 & 2.344033 & .000000 \\
\hline 5 & 6 & $\mathbf{0}$ & 1.395074 & .904152 & .000000 \\
\hline 6 & 6 & $\mathbf{0}$ & -.136338 & .803961 & .000000 \\
\hline 7 & 6 & $\mathbf{0}$ & -.649060 & -.642956 & .000000 \\
\hline 8 & 6 & $\mathbf{0}$ & -2.170956 & -.757886 & .000000 \\
\hline 9 & 16 & $\mathbf{0}$ & -2.745115 & -2.503134 & .000000 \\
\hline 10 & 8 & $\mathbf{0}$ & -2.170956 & -3.074543 & 1.253142 \\
\hline 11 & 8 & $\mathbf{0}$ & -2.170956 & -3.074543 & -1.253142 \\
\hline 12 & 8 & $\mathbf{0}$ & -4.230141 & -2.384787 & .000000 \\
\hline 13 & 1 & $\mathbf{0}$ & 5.871321 & 4.996323 & .000000 \\
\hline 14 & 1 & $\mathbf{0}$ & 5.943251 & 3.462774 & -.884213 \\
\hline 15 & 1 & $\mathbf{0}$ & 3.596803 & 4.407873 & -.877872 \\
\hline 16 & 1 & $\mathbf{0}$ & 3.853271 & 1.904677 & -.878156 \\
\hline 17 & 1 & $\mathbf{0}$ & 1.531630 & 2.877460 & -.878409 \\
\hline 18 & 1 & $\mathbf{0}$ & 1.790652 & .371642 & -.877826 \\
\hline 19 & 1 & $\mathbf{0}$ & -.532302 & 1.336434 & -.878882 \\
\hline
\end{tabular}




\begin{tabular}{|c|c|c|c|c|c|}
\hline 20 & 1 & $\mathbf{0}$ & -.265346 & -1.176562 & -.879011 \\
\hline 21 & 1 & $\mathbf{0}$ & -2.606520 & -.283694 & -.887497 \\
\hline 22 & 1 & $\mathbf{0}$ & 5.943251 & 3.462774 & .884213 \\
\hline 23 & 1 & $\mathbf{0}$ & 3.596803 & 4.407873 & .877872 \\
\hline 24 & 1 & 0 & 3.853271 & 1.904677 & .878156 \\
\hline 25 & 1 & 0 & 1.531630 & 2.877460 & .878409 \\
\hline 26 & 1 & $\mathbf{0}$ & 1.790652 & .371642 & .877826 \\
\hline 27 & 1 & $\mathbf{0}$ & -.532302 & 1.336434 & .878882 \\
\hline 28 & 1 & $\mathbf{0}$ & -.265346 & -1.176562 & .879011 \\
\hline 29 & 1 & $\mathbf{0}$ & -2.606520 & -.283694 & .887497 \\
\hline
\end{tabular}

Table 2

Mulliken atomic charges:

\begin{tabular}{|c|c|c|}
\hline & & \\
\hline C & $\mathrm{C}$ & -.439070 \\
\hline & & -.242986 \\
\hline & & -.242107 \\
\hline & & -.248066 \\
\hline & & -.246965 \\
\hline & $\mathrm{C}$ & -.248999 \\
\hline & & -.256806 \\
\hline & & -.44 \\
\hline & & 1.11 \\
\hline & O & 366 \\
\hline & $\mathbf{O}$ & 366 \\
\hline & $\mathbf{O}$ & 986 \\
\hline & H & 882 \\
\hline 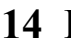 & H & 174 \\
\hline & H & 100 \\
\hline 10 & H & 340 \\
\hline- & $\mathbf{H}$ & \\
\hline 18 & H & 802 \\
\hline 19 & H & .110488 \\
\hline 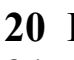 & H & .145870 \\
\hline & $\mathbf{H}$ & .131976 \\
\hline & H & .137174 \\
\hline & H & .124400 \\
\hline 241 & H & .122340 \\
\hline & H & .118244 \\
\hline 26 & H & .122802 \\
\hline 271 & H & .110488 \\
\hline & 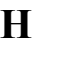 & .145870 \\
\hline & H & 131976 \\
\hline
\end{tabular}

Sum of Mulliken charges= $\mathbf{- 1 . 0 0 0 0 0}$ 
Table 3

PFOS Standard orientation:

\begin{tabular}{|c|c|c|c|c|c|}
\hline \multirow{2}{*}{$\begin{array}{l}\text { Center } \\
\text { Number }\end{array}$} & \multirow{2}{*}{\multicolumn{2}{|c|}{$\begin{array}{l}\text { Atomic } \\
\text { Number }\end{array}$}} & \multirow{2}{*}{$\begin{array}{c}\text { Atomic } \\
\text { Type }\end{array}$} & \multicolumn{2}{|c|}{ Coordinates (Angstr } \\
\hline & & & & $\mathbf{X}$ & $\mathbf{Y}$ \\
\hline 1 & 6 & $\mathbf{0}$ & .381568 & 5.414931 & .000000 \\
\hline 2 & 6 & $\mathbf{0}$ & -.469577 & 4.114634 & .000000 \\
\hline 3 & 6 & $\mathbf{0}$ & .359034 & 2.795463 & .000000 \\
\hline 4 & 6 & $\mathbf{0}$ & -.501158 & 1.490376 & .000000 \\
\hline 5 & 6 & $\mathbf{0}$ & .335452 & .167407 & .000000 \\
\hline 6 & 6 & $\mathbf{0}$ & -.524843 & -1.139896 & .000000 \\
\hline 7 & 6 & $\mathbf{0}$ & .304339 & -2.467866 & .000000 \\
\hline 8 & 6 & $\mathbf{0}$ & -.542183 & -3.767058 & .000000 \\
\hline 9 & 16 & $\mathbf{0}$ & .418038 & -5.401522 & .000000 \\
\hline 10 & 8 & $\mathbf{0}$ & 1.200815 & -5.318565 & 1.252108 \\
\hline 11 & 8 & $\mathbf{0}$ & 1.200815 & -5.318565 & -1.252108 \\
\hline 12 & 8 & $\mathbf{0}$ & -.692852 & -6.377466 & .000000 \\
\hline 13 & 9 & $\mathbf{0}$ & -.450908 & 6.467499 & .000000 \\
\hline 14 & 9 & $\mathbf{0}$ & 1.155770 & 5.485506 & -1.089736 \\
\hline 15 & 9 & $\mathbf{0}$ & -1.252232 & 4.158679 & -1.103411 \\
\hline 16 & 9 & $\mathbf{0}$ & 1.144501 & 2.813871 & -1.103617 \\
\hline 17 & 9 & $\mathbf{0}$ & -1.285495 & 1.524355 & -1.103706 \\
\hline 18 & 9 & $\mathbf{0}$ & 1.120048 & .190599 & -1.103805 \\
\hline 19 & 9 & $\mathbf{0}$ & -1.312921 & -1.094299 & -1.103309 \\
\hline 20 & 9 & $\mathbf{0}$ & 1.089738 & -2.431991 & -1.104140 \\
\hline 21 & 9 & $\mathbf{0}$ & $-\mathbf{1 . 3 4 5 8 7 7}$ & -3.719737 & -1.100819 \\
\hline 22 & 9 & $\mathbf{0}$ & 1.155770 & 5.485506 & 1.089736 \\
\hline 23 & 9 & $\mathbf{0}$ & -1.252232 & 4.158679 & 1.103411 \\
\hline 24 & 9 & $\mathbf{0}$ & 1.144501 & 2.813871 & 1.103617 \\
\hline 25 & 9 & $\mathbf{0}$ & -1.285495 & 1.524355 & 1.103706 \\
\hline 26 & 9 & $\mathbf{0}$ & 1.120048 & .190599 & 1.103805 \\
\hline 27 & 9 & $\mathbf{0}$ & -1.312921 & -1.094299 & 1.103309 \\
\hline 28 & 9 & $\mathbf{0}$ & 1.089738 & -2.431991 & 1.104140 \\
\hline 29 & 9 & $\mathbf{0}$ & -1.345877 & -3.719737 & 1.100819 \\
\hline
\end{tabular}

Table 4

Mulliken atomic charges:
1
$1 \mathrm{C} \quad \mathbf{. 7 8 3 8 7 8}$
2 C $\mathbf{. 4 8 6 8 0 9}$
$3 \mathrm{C} \quad \mathbf{. 5 3 6 8 6 2}$
4 C $\mathbf{. 5 4 3 6 1 7}$ 

5 C $\quad \mathbf{5 4 5 7 1 0}$
$\begin{array}{lll}6 & \mathrm{C} & \mathbf{5 3 1 6 8 8}\end{array}$
$\begin{array}{lll}7 & \mathrm{C} & \mathbf{5 4 8 2 3 9}\end{array}$
$\begin{array}{lll}8 & \mathrm{C} & \mathbf{3 3 4 9 5 4}\end{array}$
$\begin{array}{lll}9 & \mathrm{~S} & \mathbf{1 . 1 2 1 0 7 4}\end{array}$
$\begin{array}{llll}10 & O & -.589254\end{array}$
$\begin{array}{llll}11 & O & -.589254\end{array}$
$\begin{array}{lll}12 & \mathrm{O} & -\mathbf{. 5 8 0 6 0 5}\end{array}$
$13 \mathrm{~F}-\mathbf{- . 2 5 5 6 6 1}$
14 F -.246489
$15 \mathrm{~F}-\mathbf{- 2 7 3 0 4 5}$
16 F -.276079
17 F -.278760
$18 \mathrm{~F}-.279280$
$\begin{array}{lll}19 & F & -.283657\end{array}$
$\begin{array}{llll}20 & F & -.277319\end{array}$
$\begin{array}{llll}21 & F & -.294401\end{array}$
22 F $\quad-.246489$
23 F -.273045
24 F -.276079
25 F -.278760
26 F $-\mathbf{- 2 7 9 2 8 0}$
$\begin{array}{lll}27 & \text { F } & -.283657\end{array}$
$\begin{array}{llll}28 & F & -.277319\end{array}$
$\begin{array}{llll}29 & F & -.294401\end{array}$

Sum of Mulliken charges $=\mathbf{- 1 . 0 0 0 0 0}$

Table 5. Regression Coefficients $\left(r^{2}\right)$ and Parameters for Model Fits

\begin{tabular}{cccc}
$\mathbf{r}^{2}$ & Linear & Langmuir & Freundlich \\
\hline Geothite & 0.904 & 0.906 & 0.927 \\
OSS & 0.918 & 0.932 & 0.978 \\
High Fe Sand & 0.948 & 0.971 & 0.958 \\
Kaolinite & 0.991 & 0.937 & 0.966
\end{tabular}

\begin{tabular}{ccccccc} 
Parameters & slope & intercept & $\mathrm{a}$ & $\mathrm{b}$ & $\mathrm{K}$ & $\mathrm{n}$ \\
\hline Geothite & $1.90 \times 10^{-4}$ & $1.16 \times 10^{-4}$ & $3.75 \times 10^{-4}$ & 0.236 & $3.37 \times 10^{-4}$ & 0.555 \\
OSS & 13.5 & 29.5 & 154 & 0.488 & 50.0 & 2.19 \\
High Fe Sand & $1.42 \times 10^{-3}$ & $2.23 \times 10^{-4}$ & -0.0181 & -0.0499 & $8.73 \times 10^{-4}$ & 0.792 \\
Kaolinite & $5.49 \times 10^{-4}$ & $6.01 \times 10^{-4}$ & 0.0111 & 0.0966 & $1.18 \times 10^{-3}$ & 1.48
\end{tabular}

\footnotetext{
Note: OSS = Ottawa Sand Standard
} 


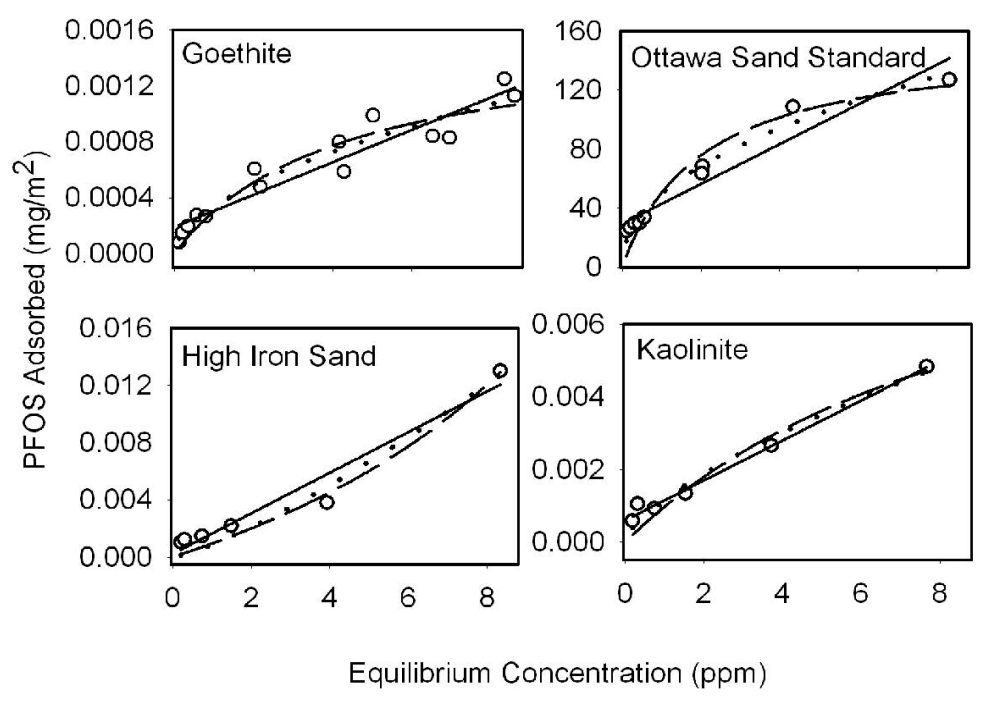

Figure 1. Isotherms: Dashed=Langmuir; Dotted=Freundlich; Solid=Linear 


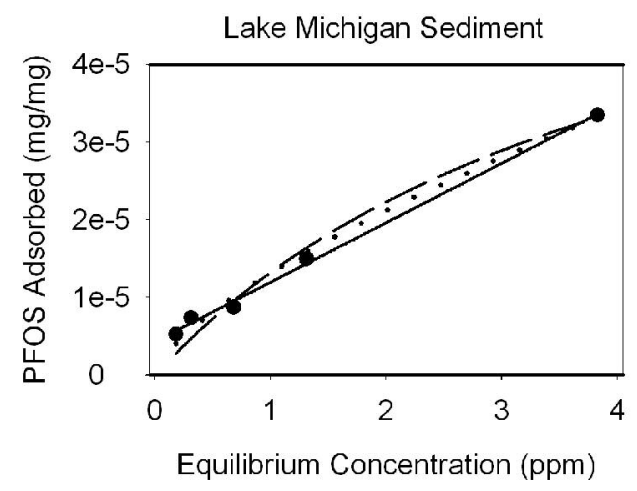

Figure 2

Figure 2. Isotherms: Dashed=Langmuir; Dotted=Freundlich; Solid=Linear 